\title{
8 Die Rolle des grammatischen Aspekts in der Ereignisenkodierung: Ein Vergleich zwischen tschechischen und russischen Lernern des Deutschen
}

\subsection{Einführung}

In diesem Kapitel wird eine Sprachproduktionsstudie vorgestellt, die sich im Fokus mit dem Vergleich von zwei slawischen Sprachen und den Aspektpräferenzen der Sprecher befasst. Dabei müssen zunächst einige theoretische Überlegungen in Bezug auf den Aspekt und die aspektuellen Werte angestellt werden, welche für die beiden untersuchten Sprachen von hoher Relevanz sind. Diese Überlegungen knüpfen direkt an die bereits in diesem Buch präsentierten theoretischen sowie empirischen Grundlagen zum Gebrauch von Aspekt in der Ereignisenkodierung an (siehe Kap. 2 - allgemeine Darstellung der Aspektforschung; Kap. 3 - konkrete Verwendung einer Aspektform im Tschechischen, Kap. 5 und 6 - empirisch-experimentelle Studien zur Aspektverwendung). In Ergänzung zu den bisherigen Ausführungen kommt in diesem Kapitel eine genauere Beschreibung der Bildungsmöglichkeiten der aspektuellen Werte im Russischen und Tschechischen hinzu, eine kritische Reflexion der in der Forschungsliteratur vorherrschenden Rezeption des sogenannten slawischen Aspekts und eine Anbindung an die Theorie von Tempus und Aspekt von Klein (1994).

Sprachen verfügen über unterschiedliche Mittel, um Zeitkonzepte auszudrücken. $\mathrm{Zu}$ den wichtigsten zählen drei verbale Kategorien: Tempus, Aspekt und Aktionsart sowie temporale Adverbiale. Jedes dieser Mittel bringt einen Teil temporaler Informationen zum Ausdruck, die jeweils mit den unterschiedlichen Teilgebieten des Sprachsystems - Lexikon, Morphologie, Syntax und Pragmatik interagieren. Üblicherweise konzentriert sich die Forschung auf die Analyse eines Teilgebietes innerhalb eines Systems (vgl. Klimonow 2000 für Aspekt und Syntax; Townsend 2001 für Aspekt und Lexikon; Israeli 2001 für Aspekt und Pragmatik; Mehlig 2001; Babko-Malaya 2003; Yanko 2003 für Aspekt und Morphologie; Zaucer 2005). In der vorliegenden Studie wird das Zusammenspiel der verschiedenen temporalen Mittel zum Ausdruck von Zeitlichkeit untersucht.

Einen besonders erhellenden Zugang bieten Analysen (sehr) fortgeschrittener Lernervarietäten. Im Rahmen dieser Arbeit wird der Frage nach ultimate attainment (Endzustand) im Zweitspracherwerb (vgl. Birdsong 1992, 2014b; Hahne 2001; Davidson/Zacks/Ferreira 2003; Singleton/Ryan 2004) nachgegangen (siehe Kap. 1, Abschn. 1.1). Untersucht werden soll, inwieweit Strategien, die 
fortgeschrittene Lerner zur Versprachlichung temporaler Ereignisse in der L2 verwenden, zielsprachig sind oder aber durch die Ausgangsprache geprägt bleiben. Den Hintergrund bildet eine Reihe von Studien, die zeigen, dass grammatikalisierte Kategorien wie Aspekt und Tempus die sprachspezifischen Muster der Ereignisenkodierung sowohl in der Erst- als auch in der Zweitsprache unmittelbar bedingen (vgl. Carroll/Lambert 2003; Carroll/von Stutterheim 1993, 2003, 2010; von Stutterheim/Carroll 2006, 2007).

Es werden zwei slawische Aspektsprachen - das Tschechische und das Russische - und eine germanische Nicht-Aspektsprache - das Deutsche - miteinander verglichen. Das Tschechische und das Russische haben im Vergleich zum Deutschen einen grundsätzlich anderen Zugang zu temporalen Einordnungen und Qualifizierungen von Ereignissen, der durch den grammatischen Aspekt gekennzeichnet ist (siehe Kap. 1, Kap. 3-5).

Der Vergleich zweier slawischer Sprachen ist insofern von Bedeutung, als Beobachtungen über den so genannten slawischen Aspekt meistens auf russischen Beispielen beruhen. Solche Beobachtungen werden dann auf andere slawische Sprachen - unter Annahme von hinreichender Ähnlichkeit der zugrundeliegenden Systeme - übertragen. Wenngleich die Aspektsysteme aller slawischen Sprachen tatsächlich große Gemeinsamkeiten aufweisen, zeigt die hier vorgestellte Untersuchung, dass es trotz typologischer Nähe erhebliche Unterschiede gibt, die dazu führen, dass Sprecher verschiedener slawischer Sprachen eine andere Versprachlichung von Einzelereignissen liefern. ${ }^{124}$ Diese wirken sich auf die Informationsselektion und Perspektivierung in der Ereignisenkodierung aus.

Im Einzelnen soll geprüft werden, inwieweit sich tschechische und russische Lerner in ihren Erwerbsverläufen der L2-Deutsch ähneln bzw. unterscheiden und ob sie die für das Deutsche typische temporale Perspektivierung übernehmen. Anders als es in der L2-Erwerbsforschung zu Aspekt und Tempus üblich ist (vgl. Robinson 1990; Andersen 1991; Andersen/Shirai 1994, 1996; Bardovi-Harlig 1992, 1994, 1997, 1999; Bardovi-Harlig/Bergström 1996; Montrul/Slabakova 2002; Slabakova/Montrul 2002; Salaberry 1997, 2000), liegt der Fokus der Studie insbesondere auf den mit dem grammatischen Aspekt verbundenen Konzeptualisierungsmustern.

124 Außer dem Russischen und dem Tschechischen werden u.a. auch das Polnische und das Bulgarische untersucht. Vorläufige Analysen deuten darauf hin, dass sich auch diese Sprachen auf subtile Weise aspektuell anders verhalten, und zwar sowohl in Bezug auf den Tempus- und Aspektgebrauch als auch mit Blick auf die Versprachlichung von Ereignisendpunkten. 


\subsection{Theoretischer Hintergrund}

\subsubsection{Zum Aspektbegriff in slawischen Sprachen}

In den vorangegangenen Kapiteln, insbesondere in Kapitel 2, wurden die terminologischen Verwirrungen im Bereich der allgemeinen sprachvergleichenden Aspektforschung bereits aufgezeigt und diskutiert. Im Hinblick auf die Markierung von Aspekt in den slawischen Sprachen bedarf es darüber hinaus noch weiterer Klärung, da die Aspektsysteme dieser Sprachen besondere Eigenschaften aufweisen: Bezüglich des Russischen und Tschechischen besteht beispielsweise eine Schwierigkeit darin, dass die aspektuellen Werte perfektiv und imperfektiv sowie die Aktionsarten durch die gleichen sprachlichen Verfahren - Präfigierung und/oder Suffigierung - gebildet werden. So kann diesbezüglich die folgende Überlegung formuliert werden:

Überlegung 1: Der aspektuelle Beitrag von Präfixen und/oder Suffixen kann grammatischer und/oder lexikalischer Art sein. Von daher müssen zwei Typen des Aspekts unterschieden werden, und zwar der grammatische und der lexikalische Aspekt. Der letztere soll als Aktionsart (und nicht etwa als Aspekt) bezeichnet werden.

In den folgenden zwei Abschnitten sollen die semantischen Funktionen des grammatischen und lexikalischen Aspekts dargestellt werden, um die erforderliche begriffliche Klarheit zu schaffen.

\subsubsection{Der grammatische Aspekt}

In der slawistischen Aspektlehre finden sich zahlreiche unterschiedliche Auffassungen zu Aspektbedeutungen bzw. -funktionen. Den meisten Ausführungen scheint in diesem Zusammenhang der Begriff der so genannten Grenze (rus. предел) gemeinsam zu sein (Vinogradov 1947; Bondarko 1971; Maslov 1978; Scheljakin 1983; Petr 1987; Schlegel 2000).

Auch in den aktuellen westeuropäischen Schulen (siehe Kap. 2, Abschn. 2.2) zeigt sich weitgehend die Tendenz, den Begriff der Grenze zu verwenden. Sasse (2002) gibt einen Überblick über rezente Aspekttheorien, die von Autoren wie Bertinetto et al. (Hgg.) (1995), Smith (1991, 1997), Bhat (1999) und Breu (2000) entwickelt wurden. Alle Autoren setzen Aspektualität (aspectuality) als übergeordnete semantische Kategorie an, die als eine Art Grenze (boundary) oder Anfangs- und Endpunkt (initial and final endpoints, vgl. Smith 1997) zu definieren ist (Sasse 2002: 201). Konzepte wie Phasen, Intervalle, telische und atelische 
Situationen lassen sich aus dem (metaphorischen) Begriff der Grenze ableiten. Dieser hat sich damit in der slawistischen wie auch in der allgemeinen Aspektforschung durchgesetzt.

In Anlehnung an die Ausführungen in Maslov (1978), Bondarko (1995) und Schlegel (2000) wird in dieser Arbeit die Annahme vertreten, dass die semantische Funktion des grammatischen Aspekts darin besteht, die Abgeschlossenheit bzw. Nicht-Abgeschlossenheit der Sachverhalte zum Ausdruck zu bringen, die sich im Erreichtsein [+END] bzw. Nicht-Erreichtsein [-END] der rechten Grenze einer Ereigniszeit äußert. Anstelle des von Schlegel vorgeschlagenen Terminus Bezugsmoment wird die Bezeichnung Situationszeit TSit verwendet, die zum hier übernommenen Beschreibungsinstrumentarium von Klein (1994) gehört.

An dieser Stelle sollen zwei Begrifflichkeiten - die Situationszeit und die Topikzeit - kurz erläutert werden, die einen wesentlichen Beitrag zum Verständnis des grammatischen Aspekts leisten. Klein (1994: 3) bezeichnet die Zeit, zu der die Situation besteht, als Situationszeit (TSit). Diese wird in der infiniten Komponente eines Satzes zum Ausdruck gebracht. Die Topikzeit (TT) entspricht der finiten Komponente der Äußerung und bezieht sich auf die Zeit, für die eine Assertion gemacht wird (ebd.: 4). Klein definiert den Aspekt als eine zeitliche Relation zwischen TT und TSit.

Ausgehend von diesen Ausführungen kann folgende Überlegung bezüglich der semantischen Funktion des grammatischen Aspekts formuliert werden (für empirische Arbeiten zu Aspektpräferenzen im Russischen und Tschechischen siehe Kap. 3, 5-6):

\section{Überlegung 2:}

(i) der perfektive Aspekt drückt das Erreichtsein der rechten Grenze aus Tschechisch: $v y$-psa(-t), Russisch: $v y$-pisa(-t') 'heraus-schreib(-en)' [+END]

(ii) der imperfektive Aspekt drückt das Nicht-Erreichtsein der rechten Grenze aus Tschechisch: vy-pis-ova(-t'), Russisch: vy-pis-yva(-t') 'heraus-schreib(-en)' [-END]

Es muss jedoch betont werden, dass dies erst die vorläufige Formulierung der Funktionen des perfektiven und imperfektiven Aspekts ist. Erst nach Abschluss der Analyse der geplanten Folgeuntersuchungen wird eine Präzisierung möglich sein. Ebenso ist die systematische Untersuchung der sprachlichen Mittel beabsichtigt, durch welche die Grenze einer Handlung angegeben wird, worüber in der gegenwärtigen Forschung noch keine klaren Erkenntnisse bestehen. 


\subsubsection{Bildung der Aspektformen}

Zum formalen Ausdruck der Kategorie Aspekt verfügen das Tschechische und das Russische über ein morphologisches System, das die Opposition zwischen imperfektiven und perfektiven Verbalformen mithilfe spezieller Morpheme kennzeichnet (siehe Kap. 1, Abschn. 1.1).

Bei den Morphemen handelt es sich vor allem um Präfixe und Suffixe. Jeder Verbalstamm kann durch Präfixe und/oder durch Suffixe semantisch und/oder grammatisch erweitert werden. Die lexikalische Ausgangsform wird in dieser Arbeit als Simplex bezeichnet. Je nachdem, in welcher Kombination die beiden aspektmarkierenden Morpheme und das Simplex zusammengesetzt sind, lassen sich drei Verbalformen unterscheiden:

(i) Simplex: Tschechisch: $p s a ́-(t)$, Russisch: pisa(-t') 'schreib(-en)'

(ii) präfigierte Verben: Tschechisch: vy-psa(-t), Russisch: vy-pisa(-t’) 'heraus-schreib(-en)'

(iii) sekundäre Imperfektiva: Tschechisch: vy-pis-ova(-t); Russisch: vy-pis-yva(-t') 'heraus-schreib(-en)'125

Zum ersten nicht-präfigierten Verbaltyp zählen in beiden Sprachen in der Regel die imperfektiven Aspektformen. Die Anzahl der Simplex-Formen, die den perfektiven Aspektformen zugeordnet werden, ist sehr gering. Präfigierung führt in aller Regel zur Bildung imperfektiver Aspektformen.

Es fällt auf, dass die präfigierten Verbalformen und die sekundären Imperfektiva die gleiche lexikalische Bedeutung aufweisen. Der Unterschied zwischen diesen zwei Verbalformen ist grammatischer und nicht lexikalischer Natur. Mit anderen Worten, durch die sekundäre Imperfektivierung ändert sich nur der grammatische Aspekt der Verben. Durch die Suffigierung werden von perfektiven Verbalstämmen die korrelativen imperfektiven Aspektformen mit den imperfektivierenden Suffixen gebildet. Im Russischen zählen dazu die Suffixe -iva-/-yva-, $-v a-,-a-/-j a-$ und im Tschechischen -(o)va. Durch diesen, von Isačenko (1982: 365) als sekundäre Imperfektivierung bezeichneten Prozess entstehen in beiden Sprachen suffixale korrelative Aspektformen. Diese sind immer imperfektiv.

125 Natürlich gibt es innerhalb der einzelnen slawischen Systeme auch Verbalformen, die weitere, für die jeweilige Einzelsprache spezifische, Morphemkombinationen aufweisen, wie dies beispielsweise fürs Tschechische mit der doppelten Suffigierung (vy-pis-ova-va(-t)) der Fall ist. Solche einzelsprachenspezifischen Morphemkombinationen bleiben an dieser Stelle unberücksichtigt, da zunächst auf die Gemeinsamkeiten und nicht auf die Kontraste der slawischen Sprachen näher eingegangen werden soll. 
Abschließend sei noch einmal darauf hingewiesen, dass die Bildung der Aspektpaare in beiden Sprachen keinen systematischen Charakter aufweist. Viele Aspektformen weisen gar keine Aspektpartner auf.

\subsubsection{Der lexikalische Aspekt}

Ergänzend zum Abschnitt 5.3.2 in Kapitel 5, in dem der Aspektgebrauch unter der Perspektive der konzeptuellen Präferenzen dargestellt wurde, wird in diesem Abschnitt die Brücke zu der Aspekt-Theorie von Klein (1994) geschlagen.

In Anlehnung an diese Theorie wird davon ausgegangen, dass der lexikalische Aspekt die inhärente Intervallstruktur einer assertierten Handlung angibt. Klein schlägt eine Klassifikation der Verben nach ihren inhärenten temporalen Eigenschaften vor, die in den traditionellen Grammatiken unter der Bezeichnung Aktionsart, aspektueller Charakter, Verbaltyp oder lexikalischer Aspekt zusammengefasst werden.

Kleins Verbklassifikation liegen die Begrifflichkeiten lexikalische Gehalte (lexical contents) und TT-Kontrast (TT-contrast) zugrunde. Unter lexikalischem Gehalt versteht er ,jenen Teil der Bedeutung eines einfachen oder zusammengesetzten Ausdrucks, der aus dem Lexikon stammt“(Klein 1992: 110).

Je nachdem, ob lexikalische Gehalte einen TT-Kontrast erlauben oder nicht, können sie unterschiedliche temporale Eigenschaften aufweisen. Den TT-Kontrast erläutert Klein mit Hilfe der folgenden beiden Beispiele:

\section{(43) Georg war müde}

(44) Georg war Marias Sohn (Klein 1992: 111)

In (1) kann man sich eine andere TT (Zeit, für die eine Assertion gemacht wird), eine TT' vorstellen. Diese bezieht sich auf die Behauptung < Georg nicht müde sein> und liegt zwei Stunden nach oder vor TT. Die TT' ist in der Behauptung (1) $<$ Georg müde sein> nicht inbegriffen. In (2) dagegen lässt sich zu keiner anderen TT' behaupten, dass Georg die Eigenschaft, Marias Sohn zu sein, nicht hatte. Während die erste Eigenschaft <Georg müde sein> in (1) temporär ist und einen TT-Kontrast ermöglicht, weist die Eigenschaft <Georg Marias Sohn sein> keine temporären Merkmale auf und erlaubt daher keinen TT-Kontrast.

Je nachdem, ob lexikalische Gehalte einen TT-Kontrast erlauben oder nicht, unterscheidet Klein (1994: 81-85) drei Typen lexikalischer Gehalte:

(a) 0 -Zustand-Gehalte ( $O$-state contents) Lexikalische Gehalte ohne TT-Kontrast - wie in (2) - werden als 0-Zustand-Gehalte bezeichnet. 
(b) 1-Zustand-Gehalte (1-state contents)

Lexikalische Gehalte mit TT-Kontrast - wie in (1) - werden 1-Zustand-Gehalte genannt.

(c) 2-Zustand-Gehalte (2-state contents)

Der dritte Verbaltyp (c) bezieht sich auf lexikalische Gehalte, die zwischen 0-Zustand und 1-Zustand liegen und den TT-Kontrast nur auf einer Seite haben. Um dies zu verdeutlichen, führt Klein folgendes Beispiel an:

\section{Caesar war tot}

Die Topikzeit TT dieser Aussage liegt in der Vergangenheit und weist einen TTKontrast zu einer TT' auf, die sich auf die vergangene Zeit bezieht, zu der Caesar noch lebte. Nach Caesars Tod gibt es keine spätere TT', für die (3) nicht gelten würde. Um dies noch mehr zu verdeutlichen, wird das Verb sterben betrachtet, z.B. Caesar starb. Der lexikalische Gehalt des Ausdrucks <Caesar sterben> beinhaltet einen Übergang vom Ausgangszustand (source state) $<$ Caesar nicht tot sein $>$ zum Zielzustand (target state) <Caesar tot sein $>$ (ebd.: 86).

Im Folgenden wird der Begriff 'Zustand', der eher statische Assoziationen hervorruft, durch den Begriff 'Zeitintervall' ersetzt. So kann folgende Überlegung festgehalten werden:

Überlegung 3: Der lexikalische Aspekt legt die inhärente Intervallstruktur einer Handlung fest, über die eine Aussage gemacht wird.

(i) 1-Zustandsverben $\Rightarrow$ ein Zeitintervall Simplex-Formen

Tschechisch - psá (-t)

Russisch - pisa $\left(-t^{\prime}\right)$

Deutsch - schreib(-en)

(ii) 2-Zustandsverben $\Rightarrow$ zwei Zeitintervalle

Präfigierte Formen

Tschechisch $-v y-p s a(-t)$

Russisch - vy-pisa $\left(-t^{\prime}\right)$

Deutsch - heraus-schreib(-en)

In den oben angeführten Beispielen tragen die Präfixe in allen drei Sprachen dazu bei, für die Assertion das zweite Zeitintervall, genauer dessen Nachzustand, auszuwählen. Kleins Verbtypologie hat gegenüber anderen Aktionsart-Typologien (vgl. Vendler 1967; Smith 1991; Bondarko 2003), die sich ständig um weitere Differenzierung von Aktionsarten in einer bestimmten Sprache bemühen, den 
Vorteil, dass sie sich auf Sprachen mit unterschiedlichem Verbalwortschatz anwenden lässt und somit auch für die Zwecke der in diesem Kapitel vorgestellten Studie geeignet ist, in der die Versprachlichung von Ereignissen im Tschechischen, Russischen und Deutschen thematisiert wird.

Auch wird hier der Unterschied zwischen grammatischem und lexikalischem Aspekt deutlich. Ein grammatischer Aspekt existiert nur in Sprachen, in denen die aspektuellen Bedeutungen grammatikalisiert sind. Der lexikalische Gehalt ist dagegen durch den lexikalischen Gehalt eines Verbs bedingt; jedes Verb weist eine Bedeutung und damit eine bestimmte inhärente Intervallstruktur auf.

\subsubsection{Morphologische und lexikalische Mittel der Endpunktmarkierung}

Räumliche Informationen können ebenfalls eine wesentliche Rolle in der Kodierung der rechten Grenze einer Situation spielen. In der Versprachlichung von Ereignissen werden oft Bewegungsverben wie gehen oder schwimmen aus dem Lexikon abgerufen. Während einfache Darstellungen durch nicht-präfigierte Bewegungsverben sich meistens auf nicht begrenzte, offene Situationen (Verlauf) beziehen, kann durch Hinzufügung lokaler Angaben oder durch Präfigierung (z.B. reingehen) die rechte Grenze einer Situation zum Ausdruck gebracht werden.

Wie bereits erwähnt, erfüllen Präfixe in slawischen Sprachen zwei wesentliche Funktionen: Zum einen dienen sie zur Markierung des grammatischen Aspekts, zum anderen verändern sie Aktionsarten durch Wortbildung (siehe Kap. 2). Letzteres bedeutet, dass Präfixe in allen untersuchten Sprachen die lexikalische Bedeutung des Verbs verändern bzw. erweitern können. In allen drei Sprachen gibt es darüber hinaus eine Reihe von Präfixen, die die Bewegung von einem Ort zum anderen und damit einen Ortswechsel klar signalisieren. In Tabelle 9 werden die wichtigsten Präfixe dieser Art dargestellt:

Tab. 9: Darstellung der wichtigsten Präfixe für die Versprachlichung eines Ortswechsels im Deutschen, Russischen und Tschechischen

\begin{tabular}{llll}
\hline Bewegung & Tschechisch & Russisch & Deutsch \\
\hline jemand verlässt einen Ort & od- & y- & weg-, fort- \\
\hline $\begin{array}{l}\text { Bewegung von irgendwoher zu einem } \\
\text { Objekt hin }\end{array}$ & při- & под-, при- & $\begin{array}{l}\text { heran-, herbei-, } \\
\text { hinzu- }\end{array}$ \\
\hline
\end{tabular}




\begin{tabular}{|c|c|c|c|}
\hline Bewegung & Tschechisch & Russisch & Deutsch \\
\hline $\begin{array}{l}\text { jemand geht in ein Gebiet hinein und } \\
\text { wieder hinaus }\end{array}$ & pro- & про- & durch-, hindurch- \\
\hline Bewegung nach oben & v-, na- & в-, на- & herauf- \\
\hline Bewegung von unten nach oben (in die Höhe) & vz- & B3-, & empor- \\
\hline Bewegung nach draußen & vy- & вы- & heraus-, hinaus- \\
\hline Bewegung nach drinnen & v- & B- & herein-, hinein- \\
\hline Bewegung nach unten & s- & $c-$ & $\begin{array}{l}\text { herab-, herunter-, } \\
\text { hinunter-, hinab- }\end{array}$ \\
\hline $\begin{array}{l}\text { Bewegung, bei der etwas sichtbar wird } \\
\text { bzw. nach vorn kommt }\end{array}$ & vy- & вы- & hervor- \\
\hline
\end{tabular}

Neben den Präfixen, die einen Ortswechsel ausdrücken, existieren auch solche, die sich diesbezüglich neutral verhalten. Im Deutschen sind hier unter anderem Präfixe wie vorbei- und entlang- zu nennen, die nichts über das Ziel einer Bewegung aussagen. Dies bedeutet, dass nicht jede Präfigierung zwangsläufig die Anvisierung der rechten Grenze einer Situation zur Folge hat.

Die räumlichen Ziele diverser Bewegungen können auch explizit durch lokale Angaben zum Ausdruck gebracht werden. Im Allgemeinen verweisen lokale Angaben auf Ausgangsorte, Zielorte oder Wege von Objektmanipulationen. Um eine präzisere Datenanalyse zu gewährleisten, müssen hier folgende vier Typen von lokalen Angaben unterschieden werden:

1. Lok: Es handelt sich um lokale Angaben im allgemeineren Sinne. Sie geben Hinweise bezüglich der räumlichen Positionen eines Objektes bzw. einer Person und werden in der Regel durch die Präpositionen in, auf (tsch. $v$, na rus. в, на) und ein Nomen im Dativ (tsch. / rus. Dativ/Präpositiv) zum Ausdruck gebracht. Beispiel: Ein Kind springt auf dem Sofa.

2. Lok $_{\mathrm{AUSG}}$ : Dieser Typ weist explizit auf den Ausgangsort einer Handlung hin. Beispiel: Eine Dose fällt vom Tisch.

3. Lok $_{\mathrm{zA}}$ : Beim dritten Typ wird das räumliche Ziel meistens mit Hilfe der Präpositionen in, auf (tsch. do, na rus. в, на) und eines Nomens im Akkusativ bzw. mit Hilfe von $z u$ (tsch. $k$ rus. $\kappa$ ) und einem Nomen im Dativ angegeben, wobei das Ziel als noch nicht erreicht interpretiert wird. Beispiel: Fünf Enten watscheln auf ein Haus zu.

4. Lok $_{\mathrm{zE}}$ : Der letzte Typ entspricht dem dritten mit dem einzigen Unterschied, dass hier das räumliche Ziel als erreicht interpretiert wird. Beispiel: Eine Dose landet auf dem Boden. 
Die angeführten Beispiele zeigen, dass nicht alle Typen von lokalen Angaben dazu beitragen, die rechte Grenze einer Situation zu kodieren. Genau genommen leisten dies nur der dritte und der vierte Typ der lokalen Angaben. Aus diesem Grund werden nur diese zwei Typen in der Datenanalyse berücksichtigt.

Die für die Auswertungen relevanten Zielangaben weisen explizit auf den Endpunkt des Zeitintervalls hin. Dieser kann je nach kontextuellen oder grammatischen Informationen vom Hörer als erreicht bzw. als nicht erreicht interpretiert werden. Die morphologische und lexikalische Endpunktmarkierung in die Datenanalyse einzubeziehen ist insofern von Bedeutung, als sich die Endpunktmarkierung in allen drei Sprachen findet. Dies bedeutet, dass hier die eventuellen Unterschiede nicht nur für die L1-, sondern auch für die L2-Daten kontrastiv dargestellt werden können.

\subsection{Fragestellung}

Generell geht es in diesem Kapitel um die Frage, wie Sprecher unterschiedlicher Sprachen Ereignisse konzeptualisieren und versprachlichen. Die zugrundeliegende These besagt, dass sprachstrukturelle Merkmale, die in gewisser Weise als gefrorene Konzeptualisierungsschemata angesehen werden können, Präferenzen für die Informationsverarbeitung implizieren. Diese Konzeptualisierungsschemata sind sprachspezifisch und beeinflussen die Präferenzen, die ein Zweitsprachlerner in einer L2 hat. Daher erweist es sich als ausgesprochen schwierig, diese Präferenzen in der Zielsprache zu erkennen (vgl. Carroll/Lambert 2003; Carroll/von Stutterheim/Nüse 2004; von Stutterheim/Nüse 2003; von Stutterheim/Carroll 2005, 2006; von Stutterheim/Lambert 2005).

Im Folgenden wird untersucht, ob Prinzipien der Informationsverteilung von den Lernern der jeweiligen Sprache erfolgreich angewendet werden oder ob Schwierigkeiten auftreten, die möglicherweise als (negativer) Transfer aus der Muttersprache erklärbar sind, und sich somit die Frage nach ultimate attainment stellt: Inwiefern sind fortgeschrittene oder sehr fortgeschrittene Lerner in der Lage, die sprachlichen Prinzipien, die die Informationsverteilung und die temporale Struktur in der L2 steuern, zu erlernen und (unter Umständen) die L1-spezifischen Selektionsmechanismen zu unterdrücken?

Es ist hier wichtig hervorzuheben, dass die Fehler, die von Lernern in diesem Bereich gemacht werden, weder grammatischer noch stilistischer Natur sind. Sie sind vielmehr auf eine nicht zielsprachige Präferenz zurückzuführen, durch die die Lerner als Nicht-Muttersprachler identifiziert werden können. Die Lerner produzieren keine grammatisch falschen Sätze, aber sie verwenden Strukturen, die von Muttersprachlern nicht verwendet werden, da sie 
den sprachspezifischen Prinzipien der zeitlichen Informationsverteilung nicht genügen.

Es kann als Stand der wissenschaftlichen Forschung gelten, dass der Aspekterwerb in slawischen L2-Sprachen mit erheblichen Schwierigkeiten verbunden ist. Das hängt u.a. damit zusammen, dass Verben slawischer Sprachen - bis auf wenige Ausnahmen - in zwei Formen existieren: in der perfektiven und in der imperfektiven. Diese zwei Formen sollen die so genannten Aspektpaare bilden (Comrie 1976; Short 1993; Bondarko 1995; Karlík 1995). Die Schwierigkeit besteht aber darin, dass weniger als die Hälfte aller Verben echte Aspektpaare bilden können (Klein 1995; Schmiedtová 2003a, 2004). So ist der Lerner stets mit der Aufgabe konfrontiert, jedes Verb doppelt lernen bzw. anwenden zu können (vgl. Schmiedtová 2004; Slabakova 2005). Ferner stellt sich die Frage, was passiert, wenn die Ausgangssprache über Kategorien verfügt, die in der Zielsprache schwächer oder gar nicht grammatikalisiert sind und für die auch nur ein beschränktes Inventar an lexikalischen Mitteln zur Verfügung steht (vgl. Bybee/Dahl 1989). Die beiden Ausgangssprachen operieren mit einem reichen und produktiven Tempus-Aspekt-System, in dem die Aspektmarkierung obligatorisch und die Opposition zwischen imperfektivem und perfektivem Aspekt grammatikalisiert ist. Die Situation in der Zielsprache ist anders: Zwar können hier aspektuelle Bedeutungen durch andere Mittel ausgedrückt werden (z.B. temporale Adverbien wie gerade), der Aspekt als solcher ist im Deutschen jedoch nicht grammatikalisiert.

Ausgehend von den bestehenden Unterschieden ließe sich vermuten, dass Lerner, deren L1 ein reichhaltiges Tempus-Aspekt-System aufweist, leichter und schneller eine Zweitsprache erwerben, die über ein weniger ausdifferenziertes und somit einfacheres System verfügt. ${ }^{126}$ Wie die Studie zeigt, ist dies nicht zwingend der Fall.

\subsection{Die Studie}

\subsubsection{Probanden}

Für die Untersuchung wurden zunächst L1-Daten erhoben. Dafür wurden insgesamt 90 Probanden aufgenommen, die drei muttersprachliche Gruppen mit jeweils dreißig tschechischen, russischen und deutschen Sprechern bildeten.

126 Als Vergleichsgröße wird Schmiedtová (2004) gewählt, wo der Erwerb der L2 Tschechisch durch deutsche und englische Muttersprachler untersucht wird. 
Alle Probanden füllten einen Fragebogen aus, in dem biografische Daten und soziale Variablen ermittelt wurden.

Dabei sollten die sozialen Variablen Alter und Bildung bei den Versuchspersonen konstant gehalten werden. Aus diesem Grund wurden für das Experiment Erwachsene im Alter von 20 bis 30 Jahren (meist Studierende, Durchschnittsalter 24,6 Jahre) herangezogen, deren Erstspracherwerb (L1) bereits abgeschlossen war. Alle L1-Daten wurden in dem jeweiligen Land erhoben (d.h. in Tschechien, Russland und Deutschland). Die Geschlechterverteilung war gleichmäßig.

In einem zweiten Schritt wurden L2-Daten erhoben, die von russischen und tschechischen Lernern des Deutschen produziert wurden. Die Daten der russischen Lerner wurden in Deutschland erhoben, während die der tschechischen in Tschechien aufgenommen wurden. Die tschechischen Probanden waren alle Studierende der Germanistik und hatten bereits eine längere Zeit in einem deutschsprachigen Land verbracht.

Ziel war es, die Untersuchung vor allem auf Basis fortgeschrittener und sehr fortgeschrittener Lerner aufzubauen, um Aussagen über die mit ultimate attainment verbundenen Fragen treffen zu können. Da in der Literatur keine einhellige Meinung dazu besteht, wodurch sich ein fortgeschrittener Lerner auszeichnet, wird im Folgenden angegeben, durch welche Kriterien diese Lernergruppe im Rahmen der vorliegenden Studie definiert wurde. ${ }^{127}$

Als fortgeschritten wurden diejenigen Lerner eingestuft, die die folgenden fünf Kriterien erfüllten, wobei die ersten zwei Kriterien als die wichtigsten zu erachten sind:

1. ausgezeichnete Sprachkenntnisse: korrekter Satzbau, Deklinations- und Verbflexionsfehler kommen kaum vor, d.h. die Lerner waren formal gesehen vollkommen unauffällig;

2. aktive Benutzung der Zweitsprache im Alltag (prozentueller Anteil nicht weniger als 70:30);

3. früher Beginn des Zweitspracherwerbs: $60 \%$ der Probanden haben Deutsch bereits in der Schule gelernt;

4. das ganze Studium verläuft in deutscher Sprache, von daher trägt der Zweitspracherwerb einen gesteuerten Charakter;

5. längerer oder längere Aufenthalt(e) in einem deutschsprachigen Land.

127 Es ist klar, dass die Auswahlkriterien nicht 'ideal' sind, um zwei homogene und somit wirklich vergleichbare Lernergruppen zusammenzustellen. Dennoch ist es von Bedeutung, diese Faktoren zu erwähnen, weil sie für den Prozess des Zweitspracherwerbes ausschlaggebend sein könnten. 
Nach Maßgabe dieser fünf Kriterien wurden für jede Sprache (Tschechisch und Russisch) jeweils 15 Lerner aufgenommen, deren Sprachproduktion in L2 Deutsch als (sehr) fortgeschritten eingestuft werden konnte. In beiden Gruppen überwog, bei ausgeglichenem Verhältnis, die Anzahl der weiblichen Lerner.

\subsubsection{Experimentablauf}

Als Stimulusmaterial dienten 40 kurze Videoclips, die alltägliche Situationen aus dem realen Leben darstellten. Es handelte sich hier um eine Online-Versprachlichung von dynamischen, singulären Situationen, die als Ereignisse bezeichnet werden (von Stutterheim 2003). Die Stimuli wurden als Videoclips in einem Durchgang dargeboten. Die Probanden wurden aufgefordert, die Fragen Čto sejčas proischodit?, Co se děje? bzw. Was passiert gerade? zu beantworten, sobald sie die Antwort aufgrund des Wahrgenommenen geben konnten. Ein Experimentdurchgang dauerte ungefähr 15 Minuten.

Für die Datenerhebungen wurden die Stimuli randomisiert, sodass sich für jede Versuchsperson eine individuelle Stimulusabfolge ergab. Zwischen den Videoclips bestanden 5-sekündige Pausen. Das akustische Signal wurde zunächst digital aufgenommen und transkribiert. Anschließend wurden die transkribierten Daten kodiert und analysiert.

\subsection{Analysebereiche und Ergebnisse}

Der Schwerpunkt des empirischen Vorhabens liegt auf der Untersuchung der Versprachlichungen der visuell dargebotenen Situationen. Die Probanden werden durch die spezielle Aufgabe des Experiments aufgefordert, die Ereignisse sprachlich zu kodieren.

Frühere Untersuchungen zum Englischen, Französischen, Deutschen, Italienischen und Spanischen haben gezeigt, dass sich diese Sprachen hinsichtlich der temporalen Strukturierung der Ereignisse erheblich voneinander unterscheiden (von Stutterheim/Carroll 2003: 367-379, 2006). Je nach Sprache können Ereignisse in vielfältiger Weise temporal strukturiert werden. Wenn es um slawische Sprachen geht, bietet sich der grammatische Aspekt an, um Unterschiede auch innerhalb der slawischen Sprachen in diesem Bereich genauer darzustellen.

Da es in der vorliegenden Untersuchung nicht nur um slawische Sprachen, sondern auch um eine germanische Nicht-Aspektsprache geht, erscheint es sinn- 
voll, weitere temporale Mittel, wie Tempus und lexikalische Mittel, zu analysieren. Diese erlauben aufzuzeigen, welche Unterschiede es in der Anwendung der temporalen Mittel gibt und welche in allen drei untersuchten Sprachen aktiv benutzt werden.

Die hier dargestellte Analyse bezieht sich auf die folgenden drei Bereiche:

1. Aspektgebrauch (nur für das Tschechische und das Russische),

2. Tempusgebrauch (alle Sprachen),

3. Markierung der Endpunkte (alle Sprachen).

Im Folgenden wird aufgezeigt, dass die temporale Strukturierung der Ereignisse deutliche sprachspezifische Merkmale aufweist, die auch in der Zweitsprachproduktion der fortgeschrittenen Lerner vorhanden und auf die jeweilige L1 zurückzuführen sind.

\subsubsection{Aspektgebrauch}

In der slawistischen Aspektforschung wurde bis heute kaum den Präferenzen Aufmerksamkeit gewidmet, die sich auf die tatsächliche Verwendung einer bestimmten morphologischen Verbalform beziehen. Das heißt, Aspektanalysen werden kaum anhand von empirisch gestützten Daten durchgeführt.

Die Ergebnisse der vorliegenden Studie zeigen, dass die Sprecher des Tschechischen und des Russischen trotz der Ähnlichkeiten in den zugrundeliegenden Aspektsystemen unterschiedliche Präferenzen für verschiedene morphologische Verbalformen in der Darstellung einer bestimmten Szene und damit eines bestimmten Situationstyps zeigen. Deutsche L1- sowie L2-Daten werden in diesem Kapitel nicht berücksichtigt, da das deutsche System keinen grammatikalisierten Aspekt besitzt.

Da die beiden slawischen Sprachen über ähnliche morphologische Mittel zur Markierung des grammatischen Aspekts verfügen, könnte man annehmen, dass ihre Sprecher auch ähnliche morphologische Verbalformen für die Darstellung der Szenen verwenden. Wie der weitere Verlauf der Arbeit jedoch zeigen wird, findet diese Annahme keine empirische Bestätigung.

Zunächst werden die Ergebnisse im Bereich des Aspektgebrauchs bezogen auf einen bestimmten Situationstyp in tschechischen und russischen Daten vorgestellt. Diese werden deskriptiv in Tabelle 10 dargestellt. 
Tab. 10: Aspektgebrauch in L1 Tschechisch und L1 Russisch

\begin{tabular}{llll}
\hline Verbalformen & Aspekt & Tschechisch & Russisch \\
\hline Simplex-Formen & imperfektiv & $\begin{array}{l}\text { häufig gebraucht, } \\
\text { egal welche Szene }\end{array}$ & $\begin{array}{l}\text { häufig gebraucht, } \\
\text { aber nur in bestimmten } \\
\text { Szenen }\end{array}$ \\
\hline Sekundäre Imperfektiva & imperfektiv & selten gebraucht & sehr häufig gebraucht \\
\hline Präfigierte Verben & perfektiv & häufig gebraucht & nur in bestimmten Szenen \\
\hline
\end{tabular}

Die Simplex-Formen, die vom grammatischen Aspekt her meistens imperfektiv sind, kommen in tschechischen Daten sehr oft vor, unabhängig von der Szene. Im Russischen werden solche Verbalformen hauptsächlich für Szenen verwendet, die nicht in Subintervalle gegliedert werden können und damit wenige Details enthalten (z.B.: Die Szene, in der ein Brief abgeschickt wird. Hier sieht man lediglich eine Hand, die einen Brief in einen Briefkasten einwirft.).

Sekundäre Imperfektiva, die vom grammatischen Aspekt her immer imperfektiv sind, werden von tschechischen Sprechern nur selten gebraucht. Dagegen kommen sie in russischen Daten sehr oft vor und zwar vorwiegend in Szenen mit Ereignissen, die sich in mehrere Subintervalle einteilen lassen (z.B.: Die Szene, in der eine junge Frau ein Glas Wasser ganz austrinkt. Man sieht sie das Glas heben, dann das Wasser trinken und schließlich das Glas wieder auf der Tischoberfläche abstellen.).

Präfigierte Verben, die vom grammatischen Aspekt her meistens perfektiv sind, kommen in tschechischen Daten sehr oft vor. In russischen Daten kommen sie vorwiegend in jenen Szenen vor, in denen eine Bewegung von Punkt A zu Punkt B als erreicht gezeigt wird (z.B. Eine Dose rollt vom Tisch und fällt auf den Boden). Während die tschechischen Sprecher das Merkmal Abgeschlossenheit in jeder auch nur potenziell abgeschlossenen Szene suchen (z.B. Zwei Frauen gehen auf ein Haus zu, wobei sie es in der Szene nicht erreichen) und diese Perspektive durch die Verwendung einer präfigiertenperfektiven Aspektform ausdrücken, verwenden die russischen Sprecher diese präfigierte perfektive Form nur für jene Szenen, in denen eine Bewegung auch tatsächlich als abgeschlossen dargestellt wird (z.B. Eine Dose rollt vom Tisch.).

Der markanteste Unterschied in der Anwendung der Aspektformen bezieht sich jedoch auf den Gebrauch der präfigierten perfektiven Präsensformen, die im Tschechischen auch für die Online-Darstellung der hier und jetzt ablaufenden Ereignisse gebraucht werden. In Beispiel (46) wird eine Szene beschrieben, in der 
eine junge Frau ein Glas Wasser austrinkt. Die Versuchsperson gebraucht die präfigierte perfektive Präsensform, während der Stimulus noch läuft. Manchmal wird in diesem Kontext das temporale Adverb jetzt verwendet.

(46) L1 tsch.

Slečna vy-pij-e vodu

Fräulein aus-trink.Perf.Präs.3.Sg Wasser

'Ein Fräulein trinkt das Wasser aus'

Im Russischen dagegen werden präfigierte perfektive Präsensformen in Verbindung mit der Hier-und-jetzt-Bedeutung nie verwendet. Diese Aspektform hat immer eine Zukunftsbedeutung (siehe auch Kap. 3 für eine detaillierte Darstellung dieses Themas).

Um die Unterschiede in der Verwendung der morphologischen Mittel zwischen den beiden Sprachsystemen zu verdeutlichen, werden hier einige Beispiele aufgeführt, die sich auf die Szene ‘Ein Brief wird eingeworfen' beziehen. In dieser Szene wird gezeigt, wie eine Person einen Brief in einen Briefkasten einwirft und sich anschließend entfernt. Der Grund für die Wahl dieses Beispiels liegt darin, dass für ihre Versprachlichung den Sprechern der beiden Sprachen alle drei Verbalformen (d.h. Simplex-Formen, sekundäre Imperfektiva und präfigierte perfektive Verben) zur Verfügung stehen. Damit haben die Sprecher beider Sprachen die gleiche Ausgangsbasis. Dies ist nicht für jede Szene möglich, da im Tschechischen die sekundären Imperfektiva nur von einer begrenzten Anzahl von Verben gebildet werden können.

Die russischen Sprecher zeigen bei der Versprachlichung der Szene folgende Präferenz:

L1 rus.

čelovek v-bras-yva-et pis’mo $v$ počtovyj jaščik

Mensch ein-werf.Impf.Präs.3.Sg Brief in Briefkasten

'Ein Mensch wirft einen Brief in den Briefkasten ein'

In Beispiel (47) verwendet der russische Sprecher ein sekundäres Imperfektivum vbrasyvaet. Tschechische Sprecher stellen dieselbe Szene typischerweise wie folgt dar:

(48) L1 tsch.

$\begin{array}{llll}\text { někdo } & v \text {-hod-i } & \text { dopis do } & \text { schránky } \\ \text { Jemand ein-werf.Perf.Präs.3.Sg Brief in } & \text { Briefkasten } \\ \text { 'Jemand wirft einen Brief in den Briefkasten ein' }\end{array}$


Wie in Beispiel (47) wird auch in Beispiel (48) im Tschechischen eine präfigierte perfektive Präsensform verwendet. Die Ergebnisse der Analyse der Aspektformen in dieser Szene werden in Tabelle 11 zusammengefasst:

Tab. 11: Aspektgebrauch in der Szene 'Ein Brief wird eingeworfen'128

\begin{tabular}{llll}
\hline Verbalformen & Aspekt & Tschechisch & Russisch \\
\hline Simplex-Formen & imperfektiv & $39 \%$ & $32 \%$ \\
\hline Sekundäre Imperfektiva & imperfektiv & $4 \%$ & $59 \%$ \\
\hline Präfigierte Verben & perfektiv & $57 \%$ & $9 \%$ \\
\hline & & $\mathrm{N}=30$ & $\mathrm{~N}=30$ \\
\hline
\end{tabular}

Wie aus Tabelle 11 ersichtlich wird, bevorzugen tschechische Sprecher perfektive Aspektformen vom Typ präfigiert für die Versprachlichung dieser Szene $\left(\chi^{2}(2)=13,4, p=0.001\right)$. Russische Probanden dagegen zeigen eine Präferenz, imperfektive Aspektformen vom Typ sekundäres Imperfektivum anzuwenden $\left(\chi^{2}(2)=11,4, p=0.003\right)$.

Es muss hier nochmals betont werden, dass den Sprechern der beiden slawischen Sprachen dieselben morphologischen Mittel zur Verfügung stehen. Wie später gezeigt wird, hat dieser Umstand direkte Auswirkungen auf den Informationsfluss und die Perspektivierung, unter welcher Ereignisse in den beiden Sprachen kodiert werden.

Der Unterschied zwischen dem Tschechischen und dem Russischen in Bezug auf die Verwendung der jeweiligen präfigierten perfektiven Präsensform wurde bisher weder kontrastiv noch für eine der Sprachen deskriptiv festgehalten.

\subsubsection{Tempusgebrauch}

Im Unterschied zum Aspektgebrauch lässt sich der Tempusgebrauch in allen fünf Probandengruppen untersuchen. Dabei wird hier Tempus in Anlehnung an Kleins Aspektanalyse als formales Mittel zum Ausdruck der zeitlichen Relation zwischen der Topikzeit TT und der Sprechzeit TU verstanden (Klein 1992: 109).

128 Die Gesamtzahl der Äußerungen ist nicht immer 30 (von 30 russischen bzw. tschechischen Sprechern). Es wurden für die Analyse nur relevante Äußerungen herangezogen (d.h. 22 russische und 23 tschechische Sprecher). Die restlichen Äußerungen wurden ausgeschlossen, da es sich entweder um Verallgemeinerungen vom Typ Ein Brief wird verschickt oder um nominale Versprachlichungen handelte. 
Was die L1-Daten betrifft, so konnte zunächst festgestellt werden, dass deutsche Muttersprachler eine ganz andere Strategie im Bereich des Tempusgebrauchs verfolgen, als dies tschechische und russische Sprecher tun. Sie verwenden in allen Darstellungen konsequent das Präsens als Haupttempusform $\left(\chi^{2}(1)=26,13, p=0.000\right)$. Im Tschechischen und Russischen dagegen ist die Verwendung der beiden Tempusformen in den relevanten Szenen gleichmäßig verteilt (Tschechisch: $\chi^{2}(1)=0,273, p=n s$; Russisch: $\left.\chi^{2}(1)=0,465, p=n s\right)$. In den nicht relevanten Szenen (z.B. homogene Aktivitäten, Zustände) unterscheiden sich die drei Sprachen im Hinblick auf Tempuswechsel kaum voneinander.

Russische und tschechische Sprecher dagegen neigen oft zum Tempus- und somit auch zwingend zum Aspektwechsel. Die Analyse zeigt, dass der Tempuswechsel $^{129}$ bei der Versprachlichung der Ereignisse in slawischen Daten keinen willkürlichen Charakter hat und ein Zusammenhang zwischen dem Tempuswechsel und einem bestimmten Situationstyp besteht. Dieser Situationstyp schließt alle Szenen mit ein, in denen eine Handlung als abgeschlossen dargestellt wird und in denen die Sprecher beider slawischer Sprachen zum perfektiven Aspekt und somit zum Präteritum greifen. Es sei hier nochmals betont, dass die Daten szenen- und nicht etwa probandenbezogen analysiert wurden.

Um die L1- und L2-Strategien zu kontrastieren, wird zunächst die exemplarische Darstellung einer bestimmten Szene aufgezeigt. In der Szene 'Ein Hund läuft ins Gewächshaus' wird gezeigt, wie ein Hund in ein Gewächshaus hineinläuft und darin verschwindet (eine Bewegung von Punkt A zu Punkt B, wobei Punkt B im Videoclip als erreicht dargestellt wird). Die Ergebnisse der Auswertungen dieser Szene sind in Tabelle 12 dargestellt.

Tab. 12: Tempusgebrauch in der Szene ‘Ein Hund läuft ins Gewächshaus’

\begin{tabular}{llll}
\hline & Wechsel zur Vergangenheit & Präsens & N \\
\hline L1 Deutsch & $3 \%$ & $97 \%$ & 30 \\
\hline L1 Tschechisch & $60 \%$ & $40 \%$ & 30 \\
\hline L1 Russisch & $43 \%$ & $57 \%$ & 30 \\
\hline L1 Tschechisch / L2 Deutsch & $41 \%$ & $59 \%$ & 15 \\
\hline L1 Russisch / L2 Deutsch & $27 \%$ & $73 \%$ & 15 \\
\hline
\end{tabular}

129 Bei Tempuswechsel - von Präsens zu Vergangenheit - wurden alle Vergangenheitsformen (Präteritum, Perfekt und Plusquamperfekt) mitgezählt. 
Es zeigt sich, dass tschechische und russische Lerner ihren muttersprachlichen Strategien folgen und auch in der L2 zum Tempuswechsel greifen. Im Gegensatz zu deutschen Muttersprachlern haben tschechische Lerner keine Präferenz für die Verwendung der Präsensform $\left(\chi^{2}(1)=0,6, p=n s\right)$. Russische Lerner benutzen die Vergangenheitsform um 20\% häufiger als tschechische Lerner und scheinen somit näher an der Zielsprache zu sein. Die Verwendung dieser Form bleibt statistisch jedoch nur ein Trend $\left(\chi^{2}(1)=3,267, p=0.071\right)$. Der quantitative Unterschied zwischen den beiden Lernergruppen lässt sich dadurch erklären, dass die russischen mehr fortgeschritten als die tschechischen Sprecher sind. Schauen wir uns einige konkrete Beispiele in allen fünf Gruppen an.

L1 tsch.

Pejsek v-běh-nu-l

do skleníku $\leftarrow$

Hauptstruktur

Hund hinein-lauf.Perf.Prt.Sg.m in Gewächshaus im Präteritum

'Ein Hund lief in ein/das Gewächshaus hinein'

Das tschechische Beispiel besteht aus einer Äußerung, die mit Hilfe des perfektiven Präteritums gebildet wurde und eindeutig die Hauptstrukturinformationen liefert. Mit Hauptstruktur ist die Aussage gemeint, die auf die Frage Was passiert? eine direkte Antwort liefert. Durch die präfigierte Verbalform vbĕhnul wurde das Erreichen des räumlichen Ziels skleníku kodiert. Unter Tempuswechsel wird die Tatsache verstanden, dass die Mehrheit der Äußerungen im Präsens verbalisiert wurde, d.h. es wird Bezug genommen auf den Wechsel zwischen verschiedenen Situationstypen und nicht etwa auf den Wechsel innerhalb einer Szenenbeschreibung.

In ähnlicher Weise wird diese Szene von russischen Sprechern versprachlicht, wie man Beispiel (50) entnehmen kann:

L1 rus.

Sobaka za-beža-la

$v$ teplicu $\leftarrow$

Hund hinein-lauf.Perf.Prt.S.f in Gewächshaus

Hauptstruktur

'Ein Hund lief in ein/das Gewächshaus rein'

In Beispiel (50) wird ebenfalls das perfektive Präteritum für die Enkodierung der Situation benutzt. Sogar die syntaktische Struktur der russischen Äußerung stimmt mit der tschechischen exakt überein.

Deutsche Sprecher dagegen stellen die gleiche Szene meistens im Präsens dar. Wenn überhaupt ein Tempuswechsel stattfindet (3\%, siehe Tab. 12), dann meistens in Nebenstrukturäußerungen (sprich Kommentare und attributive Nebensätze etc.). Diese Tendenz wird in Beispiel (51) veranschaulicht. 
(51) L1 dt.

Ein Hund läuft in ein Gewächshaus, $\quad \leftarrow$ Hauptstruktur im Präsens dessen Tür offen stand

$\leftarrow$ Nebenstruktur im Präteritum

Wie aus Beispiel (51) ersichtlich wird, benutzt der Proband in der ersten Äußerung das Präsens und wechselt in der darauffolgenden Äußerung zum Präteritum. $\mathrm{Zu}$ beachten ist, dass die zweite Äußerung eine Art Beschreibung enthält, die normalerweise einen festen Bestandteil der Nebenstruktur bildet. Es wird im Rahmen dieser Arbeit angenommen, dass das der Grund ist, warum hier überhaupt ein Tempuswechsel stattfindet.

Die Lerner bilden zwar in ihrer L2 Deutsch grammatikalisch korrekte Sätze (d.h. formal unauffällige), verwenden dafür jedoch in ca. einem Drittel aller Äußerungen die Vergangenheitsform, die von den Muttersprachlern für die Darstellung dieser Szene eben nicht bevorzugt wird (siehe auch Kap. 1, Abschn. 1.1 für die Diskussion der sogenannten formal accuracy).

L1 tsch. / L2 dt.

Ein Hund ist in ein Glashaus gegangen

$\leftarrow$ Hauptstruktur im Perfekt

(53) L1 rus. / L2 dt.

Ein Hund ist in so ein Glashaus reingegangen $\leftarrow$ Hauptstruktur im Perfekt

Die Beispiele (52) und (53) unterscheiden sich kaum voneinander, außer dass der russische Lerner eine präfigierte Verbalform anwendet und der tschechische nicht. Beide verwenden das Perfekt und nicht, wie die zielsprachige Präferenz es vorgibt, das Präsens.

Zusammenfassend lässt sich Folgendes festhalten: Tschechische und russische Lerner des Deutschen folgen, wenn sie die Vergangenheitsformen verwenden, eindeutig ihren L1-Präferenzen, um die Abgeschlossenheit eines Ereignisses oder den Zustandswechsel einer Situation zum Ausdruck zu bringen. Dieses Ergebnis legt die Annahme nahe, dass die Lerner das Fehlen des grammatischen Aspekts im Deutschen durch nicht zielsprachigen Tempusgebrauch zu kompensieren versuchen.

\subsubsection{Endpunkte}

Da für die Versprachlichung der in den Videoclips gezeigten Situationen diverse Bewegungen und Handlungen enkodiert werden müssen, können auch räumliche Ziele - im Folgenden Endpunkte genannt - mit angegeben werden. Solche 
Endpunkte können entweder lexikalisch durch lokale Angaben (z.B. räumliche Adjunkte) oder morphologisch durch Präfixe (zum Beispiel hineinlaufen) ausgedrückt werden.

Frühere Untersuchungen haben gezeigt, dass sich Sprachen erheblich im Hinblick auf die Enkodierung der Endpunkte unterscheiden (Carroll/Lambert 2003; von Stutterheim/Lambert 2005). In dieser Studie wurden die Szenen analysiert, die für die Enkodierung der Endpunkte relevant sind. Die ausgewählten Szenen lassen sich inhaltlich in zwei Gruppen unterteilen. Zur ersten Gruppe zählen Szenen mit jenen Bewegungsereignissen, in denen kein Erreichen eines möglichen Endpunkts gezeigt wird [-END]. Die zweite Gruppe umfasst gleichfalls Szenen mit Bewegungsereignissen, in denen der Endpunkt als erreicht [+END] dargestellt wird.

Lokale Angaben und Präfixe wurden separat ausgewertet und die Ergebnisse später miteinander verglichen. Äußerungen, in denen lokale Angaben zusammen mit Präfixen zur Endpunktkodierung verwendet wurden, wurden nicht separat ausgezählt. Es wird beabsichtigt, dies in späteren Analysen mit einer größeren Probandenanzahl durchzuführen.

Als erstes werden die Ergebnisse der Enkodierung der Endpunkte durch lokale Angaben in muttersprachlichen L1- und L2-Lernerdaten behandelt. Diese sind in Tabelle 13 zusammengefasst:

Tab. 13: Enkodierung der Endpunkte in L1- und L2-Daten

\begin{tabular}{lcllll}
\hline Szene & DT. L1 & TSCH. L1 & RUS. L1 & $\begin{array}{l}\text { TSCH. L1/ } \\
\text { DT. L2 }\end{array}$ & $\begin{array}{l}\text { RUS. L1/ } \\
\text { DT. L2 }\end{array}$ \\
\hline 1. Nonnen [-END] & $30 \%$ & $63 \%$ & $30 \%$ & $65 \%$ & $40 \%$ \\
\hline 2. Zug kommt an [-END] & $63 \%$ & $80 \%$ & $34 \%$ & $77 \%$ & $47 \%$ \\
\hline 3. Kutsche [-END] & $47 \%$ & $50 \%$ & $10 \%$ & $71 \%$ & $27 \%$ \\
\hline 4. Brief einwerfen [+END] & $97 \%$ & $83 \%$ & $77 \%$ & $88 \%$ & $60 \%$ \\
\hline 5. Gewächshaus [+END] & $100 \%$ & $93 \%$ & $93 \%$ & $88 \%$ & $100 \%$ \\
\hline 6. Runterspringen [+END] & $90 \%$ & $77 \%$ & $63 \%$ & $53 \%$ & $60 \%$ \\
\hline
\end{tabular}

Was die L1-Daten betrifft, so unterscheiden sich tschechische Muttersprachler von den beiden anderen muttersprachlichen Gruppen vor allem dadurch, dass sie bedeutend mehr lokale Angaben für die Darstellung der kritischen Szenen 
heranziehen, in denen der Endpunkt als nicht erreicht [-END] gezeigt wird $\left(\chi^{2}(2)=16, p=0.000\right)$, als dies deutsche und russische Sprecher tun.

Entgegen der Erwartung, dass die Sprecher der beiden slawischen Sprachen bezüglich der Endpunktmarkierung eine gemeinsame Strategie verfolgen, stellt sich heraus, dass die tschechischen Muttersprachler anders vorgehen: Endpunkte werden in allen relevanten Szenen in der Mehrheit der Äußerungen verbalisiert. Die russischen Muttersprachler dagegen scheinen in diesem Bereich eine andere Vorgehensweise zu haben: Sie erwähnen erst dann Endpunkte, wenn diese in einem Clip auch tatsächlich als erreicht gezeigt werden. Deutsche Muttersprachler erwähnen in den Szenen 2 und 3 zwar mehr Endpunkte als russische Muttersprachler, aber nicht mehr als tschechische. Für Szenen, in denen der Endpunkt als erreicht [+END] dargestellt wird, wurden für keine der L1-Sprachen statistisch relevante Unterschiede festgestellt $\left(\chi^{2}(2)=1,69, p=n s\right)$.

Diese Präferenzen sollen nun anhand einiger Beispiele veranschaulicht werden. Sie beziehen sich auf die Szene 'Nonnen', in der folgende Situation dargestellt wird: Zwei schwarz gekleidete Frauen (Nonnen) gehen eine Landstraße entlang und unterhalten sich. Am Ende dieser Straße sind ein größeres Gebäude, das ein Kloster sein könnte, und ein paar kleinere Häuser zu sehen. In der Szene wird kein Endpunkt erreicht [-END].

L1 dt.

Zwei Nonnen gehen eine Landstraße entlang

Beispiel (10) L1 rus.

Dve monaški idut po doroge

Zwei Nonnen geh.Impf.Präs.3.Pl durch Straße

'Zwei Nonnen gehen eine Straße entlang'

Beispiel (54) und (55) zeichnen sich durch das Fehlen jeglicher Zielangabe aus und stellen prototypische Äußerungen für die Sprecher des Russischen und des Deutschen dar. Tschechische Sprecher erwähnen interessanterweise in der Mehrheit der Äußerungen Zielangaben und gehen dabei, wie in Beispiel (55) gezeigt, vor:

(55) L1 tsch.

Dvĕ jeptišky jdou po cestě $k$ nějakému stavení

Zwei Nonnen geh.Impf.Präs.3.Pl auf einem Weg zu einem Gebäude

'Zwei Nonnen gehen eine Straße entlang zu einem Gebäude'

Durch die Verwendung der lokalen Angabe $k$ nějakému stavení wird eine rechte Grenze im Zeitintervall des Gehens anvisiert, die in den Beispielen (54) und (55) völlig ausgeblendet wird. Und was machen die Lerner? 
(56)

L1 rus. / L2 dt.

Ich sehe zwei Nonnen, die einen Weg entlanggehen

(57) L1 tsch. / L2 dt.

Zwei Nonnen gehen zu einem Kloster

Wie aus den Beispielen hervorgeht, folgen Lerner beider Gruppen den L1-Mustern, indem sie lokale Angaben entweder weglassen - das russische Muster oder zu häufig erwähnen - das tschechische Muster.

Als nächstes wurden die Ergebnisse der Auszählungen der beiden Vorgehensweisen in der Enkodierung der Endpunkte (durch lokale Angaben und Präfixe) miteinander verglichen. Diese werden in Tabelle 14 dargestellt. Die Zahlen in der Tabelle beziehen sich auf die Szene 'Ein Brief wird eingeworfen', die bereits in Beispiel (47) diskutiert wurde und für diesen Zweck repräsentativ ist.

Die Äußerungen, in denen eine Simplex-Form mit einer lokalen Angabe (2. Spalte in Tab. 14) vorkommt, wurden separat von den Äußerungen gezählt, in denen präfigierte Verben mit oder ohne lokale Angaben (3. Spalte in Tab. 14) verwendet werden.

Tab. 14: Endpunkte in der Szene 'Ein Brief wird eingeworfen'

\begin{tabular}{lllll}
\hline & $\begin{array}{l}\text { Lokale } \\
\text { Angaben }\end{array}$ & $\begin{array}{l}\text { Präfixe } \\
\text { I Lokale Angaben }\end{array}$ & N & \\
\hline L1 Deutsch & $73 \%$ & $27 \%$ & 30 & \\
\hline L1 Tschechisch & $60 \%$ & $40 \%$ & 30 & \\
\hline L1 Russisch & $23 \%$ & $77 \%$ & 30 & Präfix-Strategie \\
\hline L2 Russisch/Deutsch & $47 \%$ & $53 \%$ & 15 & \\
\hline L2 Tschechisch/Deutsch & $\mathbf{9 3 \%}$ & $7 \%$ & 15 & Endpunkt-Strategie \\
\hline
\end{tabular}

Bei der Betrachtung der L1-Daten fällt sofort auf, dass russische Sprecher eine deutliche Präferenz für Präfixe zeigen $\left(\chi^{2}(1)=8,53, p=0.003\right)$, während deutsche Sprecher überwiegend lokale Angaben zum Ausdruck der Endpunkte verwenden $\left(\chi^{2}(1)=6,53, p=0.011\right)$. Tschechische Muttersprachler benutzen sowohl lokale Angaben als auch Präfixe, wenn sie in ihrer Muttersprache Endpunkte markieren $\left(\chi^{2}(1)=1,2, p=n s\right)$.

Wenn man die zwei Lernergruppen miteinander vergleicht, zeigt sich, dass tschechische Lerner Endpunkte durch lokale Angaben markieren und somit eine klare Präferenz zeigen $\left(\chi^{2}(1)=11,27, p=0.001\right)$. Wie bereits erwähnt, werden im Rus- 
sischen zur Endpunktmarkierung Präfixe verwendet. Diese Präferenz schlägt sich bei den russischen Lernern nieder, indem sie a) der deutschen Strategie, Endpunkte durch lokale Angaben zu markieren, nicht so klar folgen und b) sich von den tschechischen Lernern des Deutschen signifikant unterscheiden $\left(\chi^{2}(1)=7,78, p=0.005\right)$. Mit anderen Worten: Russische Lerner in L2 Deutsch stehen in einem Konflikt zwischen ihrer muttersprachlichen Strategie und der der Zielsprache. Dies führt dazu, dass sie in L2 Deutsch keine klare Präferenz mehr zeigen $\left(\chi^{2}(1)=0,06, p=n s\right)$. Aus diesen Ergebnissen lässt sich ableiten, dass die Lerner bei der Markierung der Endpunkte unterschiedlich vorgehen. In diesen Vorgehensweisen spiegeln sich die muttersprachlichen Tendenzen wider. Dies wird anhand eines Vergleichs der folgenden drei Beispiele verdeutlicht:

(58) L1 dt.

Jemand wirft einen Brief in einen Briefkasten

(59) L2 rus. / dt.

Eine Person wirft einen Umschlag in Briefkasten rein

(60) L2 tsch. / dt.

Ein Mann wirft einen Brief in einen Briefkasten

Der russische Muttersprachler verwendet in Beispiel (59) die präfigierte Form reinwerfen. Deutsche Sprecher wenden in 30\% der Äußerungen ebenfalls präfigierte Formen an, sie bevorzugen jedoch die Darstellungsweise wie in Beispiel (58), also die nicht-präfigierte Form. Tschechische Muttersprachler folgen einem ähnlichen Muster wie die deutschen Muttersprachler und wenden Präfixe in nur 37\% der Äußerungen an. In allen anderen Fällen bedienen sie sich einer lokalen Angabe, um einen Endpunkt zu enkodieren.

Was passiert in den Szenen, in denen die Präfixe in der Zielsprache typischerweise nicht verwendet werden? Beispielsweise in einer Szene, in der gezeigt wird, wie ein Baum gefällt wird. Wie die Analyse dieser Szene ergibt, verwenden auch hier russische Lerner Präfixe nicht so, wie dies deutsche Muttersprachler tun würden.

Tab. 15: Präfixe als Endpunkte in russischen L2-Daten in der Szene 'Baum fällen’

\begin{tabular}{lll}
\hline & L1 Deutsch & L2 Russisch/Deutsch \\
\hline$(+)$ präfigiert & $33 \%$ & $87 \%$ \\
\hline$(-)$ präfigiert & $67 \%$ & $13 \%$ \\
\hline & $\mathrm{N}=30$ & $\mathrm{~N}=15$ \\
\hline
\end{tabular}


Wie aus der Tabelle 15 ersichtlich wird, verwenden 87\% der russischen Lerner in dieser Szene präfigierte Verbformen, wohingegen ein Drittel der Deutschmuttersprachler zu präfigierten Verbalformen wie umsägen, absägen, abschneiden greift. In den Beispielen (61) und (62) werden diese Präferenzen illustriert:

(61) L1 dt.

Ein Waldarbeiter fällt einen Baum mit einer Motorsäge

(62) L2 rus. / dt.

Ein Mann sägt einen Baum ab

Es ist sicherlich nicht ungrammatisch, absägen zu verwenden - zwei deutsche Sprecher tun das auch -, aber eben untypisch für den zielsprachigen Gebrauch. Die Ergebnisse bestätigen die Annahme, dass es sich hier um eine muttersprachliche Präfix-Strategie handelt.

Zusammenfassend lässt sich festhalten, dass die Enkodierung der Endpunkte einen unterschiedlichen Häufigkeitsgrad in allen drei untersuchten Sprachen erfährt. Wenn man allerdings andere Sprachen in den Vergleich einbezieht, die kaum Endpunkte für die Enkodierung der Ereignisse benennen, wie dies beispielsweise für das Englische der Fall ist (vgl. Schmiedtová 2013b), erscheint der Unterschied zwischen russischen und deutschen Sprechern nicht wesentlich, da die Zahlen nicht so sehr auseinander liegen. Das interessante Ergebnis hier ist, dass das Russische und das Tschechische - trotz der typologischen Nähe - in dieser Hinsicht divergieren.

\subsection{Fazit und Diskussion}

Die Ergebnisse zeigen, dass das Tschechische und das Russische - obwohl beide Sprachen strukturell über dasselbe Ausdrucksinventar in den hier relevanten Bereichen grammatischer Aspektkennzeichnungen verfügen - unterschiedliche Verwendungsmuster dieser Formen aufweisen. Trotz der typologischen Nähe gehen tschechische und russische Muttersprachler bei der Enkodierung von temporalen Ereignissen anders vor: Die tschechischen Muttersprachler legen ihren Aufmerksamkeitsfokus auf das Erreichen der rechten Grenze eines Zeitintervalls und bedienen sich, soweit es das sprachliche System und der Stimulus möglich machen, der präfigierten perfektiven Form. Diese wird auch im Präsens (Hierund-jetzt-Interpretation) gebraucht. Die russischen Muttersprachler dagegen fokussieren den unmittelbar vor der rechten Grenze liegenden Zeitabschnitt und bringen diese Bedeutung mit Hilfe der sekundären Imperfektiva zum Ausdruck. 
Somit weisen diese zwei Aspektformen - die präfigierte perfektive Form und das sekundäre Imperfektivum - auf zwei unterschiedliche psycholinguistische Korrelate hin: Die perfektive Form ermöglicht den Zugriff auf die rechte Grenze, während die sekundären Imperfektiva auf das Zeitintervall vor dem Erreichen der rechten Grenze zugreifen. D.h. die tschechischen Muttersprachler zeigen eine starke Präferenz, Ereignisse in ihrer Abgeschlossenheit zu versprachlichen (und zwar auch dann, wenn das Ereignis im Videoclip nicht zu Ende geht), während die russischen Muttersprachler die Versprachlichung der mittleren Phase eines Ereignisses bevorzugen.

Diese Präferenzen schlagen sich auch in der Sprachproduktion der fortgeschrittenen L2-Lerner nieder. Beide Gruppen neigen dazu, den im Deutschen fehlenden grammatischen Aspekt mit anderen sprachlichen Mitteln auszudrücken. Dies wird im Tempusgebrauch beider Lernergruppen dann deutlich, wenn sie von dem zielsprachigen Muster zugunsten ihrer L1-Präferenzen abweichen.

Im Bereich der Versprachlichung von Endpunkten wurde gezeigt, dass die Vorgehensweisen der tschechischen und der russischen Muttersprachler divergieren: Die tschechischen Muttersprachler erwähnen Endpunkte in Szenen auch dann, wenn sie nicht als erreicht dargestellt werden [-END]. Dies geschieht viel häufiger als bei russischen und sogar auch deutschen Muttersprachlern. Interessanterweise fallen die deutschen und die tschechischen Sprecher in dieser Hinsicht in ein Cluster. Diese Präferenzen werden in den L2-Daten wiedergefunden: Sowohl tschechische als auch russische Lerner bleiben ihren L1-Präferenzen verhaftet. Dies wird besonders deutlich, wenn man sich die sprachlichen Mittel anschaut, die für die Endpunktmarkierung durch Lerner in L2 Deutsch verwendet werden. Der russische Lerner des Deutschen bevorzugt - so wie in der Ausgangssprache Russisch - die Präfixstrategie, der tschechische Lerner greift auf die in seiner L1 verankerten Strategie zurück und versprachlicht Endpunkte mittels lokaler Adjunkte.

Die Ergebnisse zeigen, dass die Konzeptualisierungsschemata, die in der Enkodierung von temporalen Ereignissen verschiedene Präferenzen für die Informationsverarbeitung setzen, einen sprachspezifischen Charakter aufweisen. Im Hinblick auf die (sehr) fortgeschrittenen Lerner, die an dieser Studie teilgenommen haben, lässt sich klar feststellen, dass es für sie so gut wie unmöglich war, die Präferenzen der Informationsverarbeitung und -selektion zielsprachig zu verwenden. Demzufolge muss die Frage nach ultimate attainment wie folgt beantwortet werden: Auch (sehr) fortgeschrittene Lerner, deren Sprachproduktion formal und grammatisch als muttersprachlich bezeichnet werden kann, fallen im Bereich temporaler Perspektivierung von Ereignissen als Nicht-Muttersprachler auf. Mit anderen Worten, sie bedienen sich auch in der L2 ihrer L1-Präferenzen. 
Zum Abschluss soll eine der Ausgangsfragen unter Berücksichtigung der im zuvor dargestellten Ergebnisse beantwortet werden: Ist der Erwerb einer Zielsprache, die über ein weniger ausdifferenziertes System als die Ausgangssprache verfügt, schneller/einfacher zu erlernen? Die vorliegende Studie beantwortet diese Frage mit Nein: Auch near-native-Sprecher drücken in der Zielsprache Strukturen aus, die in der L2 so nicht existieren. Dabei stützen sie sich auf entsprechende L1-Strukturen, die einen hohen Grammatikalisierungsgrad aufweisen und in der Ausgangssprache obligatorisch sind (z.B. der grammatische Aspekt im Tschechischen und Russischen). Diese Instanzen des negativen Transfers haben direkte Implikationen für die Konzeptualisierung von Ereignissen in der Zielsprache.

\subsection{Zusammenfassung}

Die Ergebnisse der in diesem Kapitel vorgestellten L2-Studie weisen auf die Beständigkeit der L1-Präferenzen und ihren Einfluss auf den Erwerb und Gebrauch einer Zweitsprache hin. Darüber hinaus wird gezeigt, dass russische und tschechische L2-Lerner bei der Ereignisenkodierung im Deutschen unterschiedliche Konzeptualisierungsschemata verwenden, die sich auf die L1-spezifischen Präferenzen zurückführen lassen. Im Rückschluss unterstützen die L2-Daten die Befunde zu den muttersprachlichen Präferenzen und leisten somit einen wichtigen Beitrag zur Sprache-und-Kognitions-Debatte. 
